\title{
Not a String, not a Tangle, not an Aneurysm
}

\section{Emerging Pattern of Large Parenchymal Bleeding in Younger Patients Associated with Abnormal Vessels on Imaging}

\author{
Patrick Nicholson ${ }^{1}$ (D) - Meah Gao $\cdot$ Ivan Radovanovic ${ }^{2}$ Vitor Mendes-Pereira ${ }^{1,2}$ - Mojgan Hodaie ${ }^{2}$. \\ Aleksandra Pikula ${ }^{3} \cdot$ Timo Krings ${ }^{1,2}$
}

Received: 3 June 2020 / Accepted: 28 July 2020 / Published online: 19 August 2020

(c) Springer-Verlag GmbH Germany, part of Springer Nature 2020

\begin{abstract}
Purpose Intracerebral hemorrhage (ICH) accounts for up to $20 \%$ of all strokes, and there is a high rate of associated morbidity and mortality. Computed tomography (CT) findings, such as a spot sign have been shown to be an independent predictor of poor outcome. We have recently encountered a succession of ICH patients who presented with a peculiar imaging finding, which we term the spot on a string sign. This is a rare imaging finding, and interestingly, all these patients presented to our institution over the last few weeks.

Methods This was a single high-volume center series of patients who presented to our institution between 1 April and 21 May 2020. All patients underwent initial non-contrast CT brain and subsequent CT angiography (CTA). We also present laboratory and clinical data. Our primary measure was the presence of the spot on a string sign on the CTA. We also report the clinical course of these patients.

Results In this study seven large-volume ICH patients with this imaging sign were identified, with a median age of 48 years (range 30-68 years). All had tested negative for coronavirus disease 19 (COVID-19).

Conclusion We have described an unusual imaging finding in a cohort of younger patients with large-volume ICH, all of whom presented in a 2-month period to a high-volume neurovascular unit. The cause of these ICH presentations and associated imaging findings are unclear, but we encourage other clinicians to be aware of and vigilant for this rare phenomenon, especially in younger patients with such a bleeding pattern.
\end{abstract}

Keywords Intracranial hemorrhage $\cdot \mathrm{ICH} \cdot$ Stroke $\cdot$ Hemorrhage $\cdot$ Vascular

\section{Introduction}

Nontraumatic intracerebral hemorrhage (ICH) accounts for up to $20 \%$ of all strokes [1]. A computed tomography angiogram (CTA) is often performed to exclude an underlying vascular pathology (e.g. aneurysm, arteriovenous malformation $[\mathrm{AVM}])$. The CTA spot sign is defined as a unifocal/multifocal area of contrast enhancement within the hematoma and is associated with poor clinical outcomes [2]. By definition, a spot sign should not be connected to a blood vessel as it is supposed to represent rupture of parenchymal microaneurysms. These were initially described in 1868 by Charcot and Bouchard and are reported to measure up to $2 \mathrm{~mm}$ in size [3]. One group has described a spot and tail sign in 15 patients with putaminal hemorrhage [4]. We have recently noticed a similar imaging pattern emerge in a series 


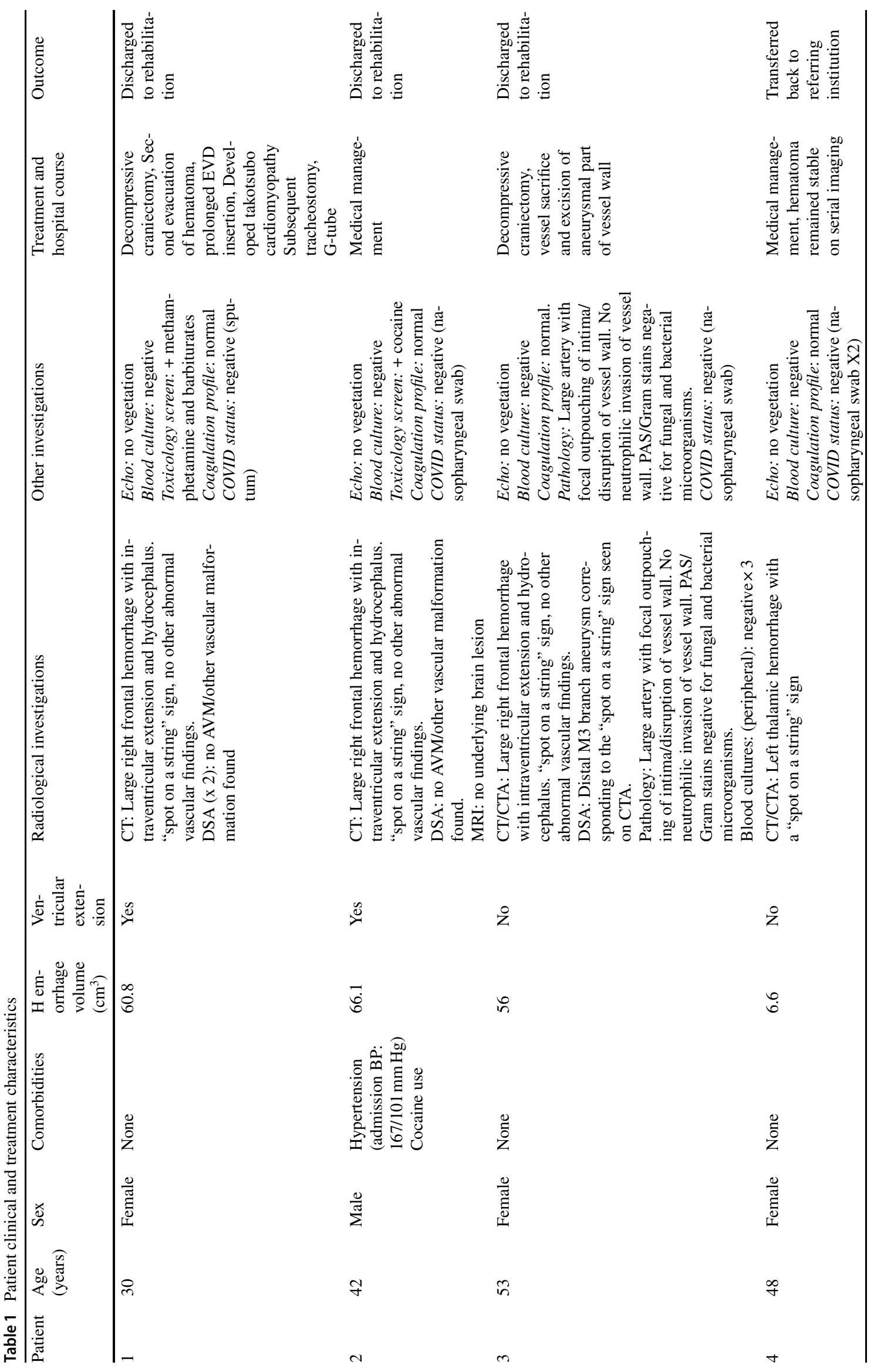




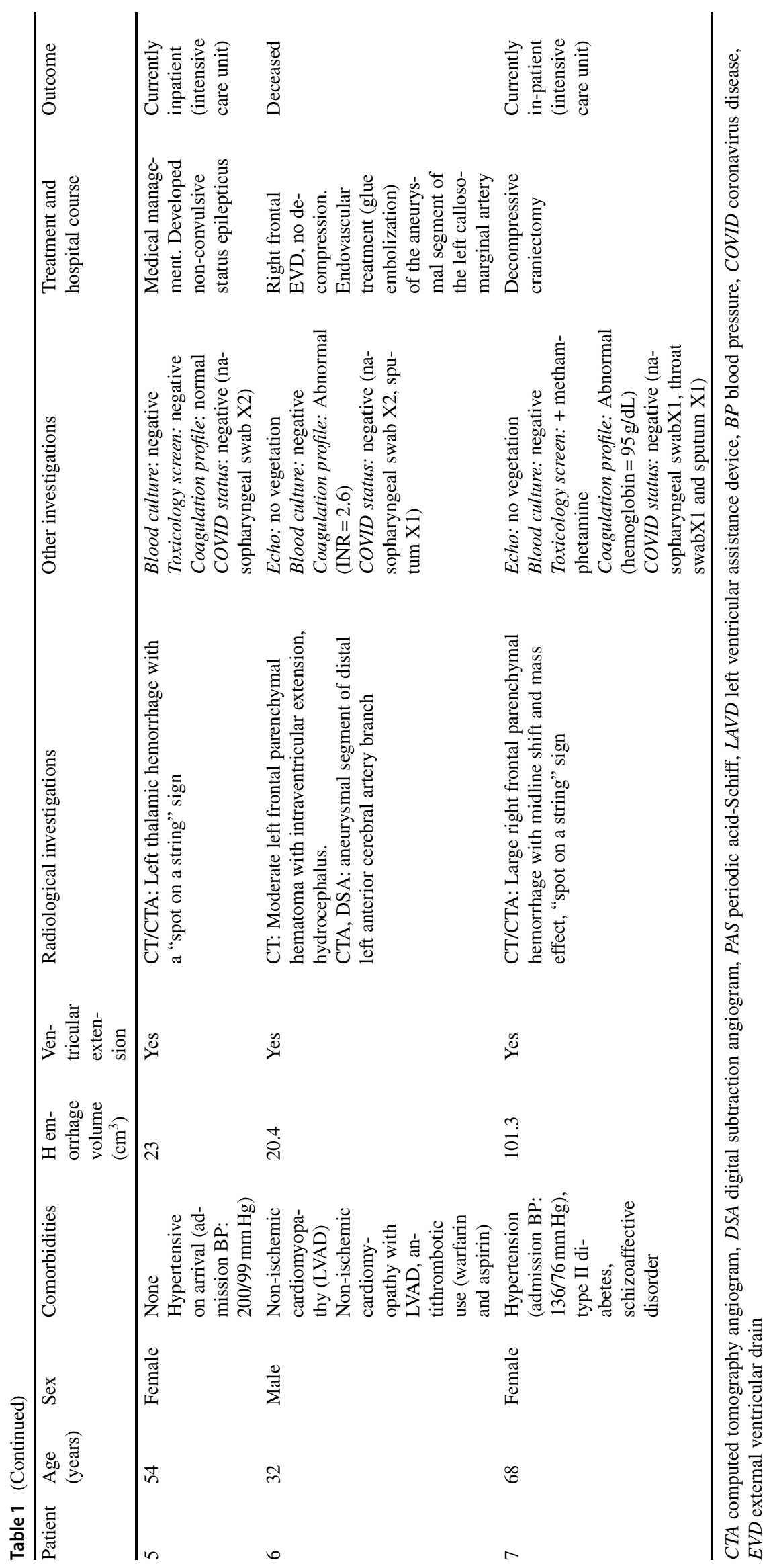


of younger patients with no underlying comorbidities, who presented to our institution with a new ICH. These patients all presented with a peculiar spot-sign which was connected to a vessel (lenticulostriate/choroidal/leptomeningeal perforators). We call this imaging finding a spot on a string sign. In this article, we describe the imaging and clinical features of these patients and discuss possible causes.

\section{Methods}

A prospectively collected database was maintained of patients who presented to our center with new primary ICH, and no history of trauma/active infective endocarditis or other potential causes. Patient clinical demographics were recorded. All images was re-read by a fellowship-trained neuroradiologist. The hematoma volume was measured using the well-established ABC/2 [5] method.

\section{Results}

A total of seven patients were identified (Table 1) with a spot on a string sign on CTA. Median age was 48 years (range 30-68 years), of which 2 had a history of hypertension while another (patient 5) was hypertensive on arrival and 3 had positive toxicology screens for either cocaine or methamphetamines. All were COVID-negative. Of the patients one underwent a multiphase study, and the "spot" did not expand on the delayed phases, six had negative echocardiograms, and all had serial negative blood cultures. Of the patients three underwent medical management, two underwent decompressive craniotomy and hematoma removal, while one underwent emergency embolization of the aneurysmal segment of the vessel. There was no extravasation of contrast medium present in the two patients who underwent digital subtraction angiograms. One underwent craniotomy, hematoma removal and resection of the abnormal vessel. Pathology in this case showed an aneurysmal
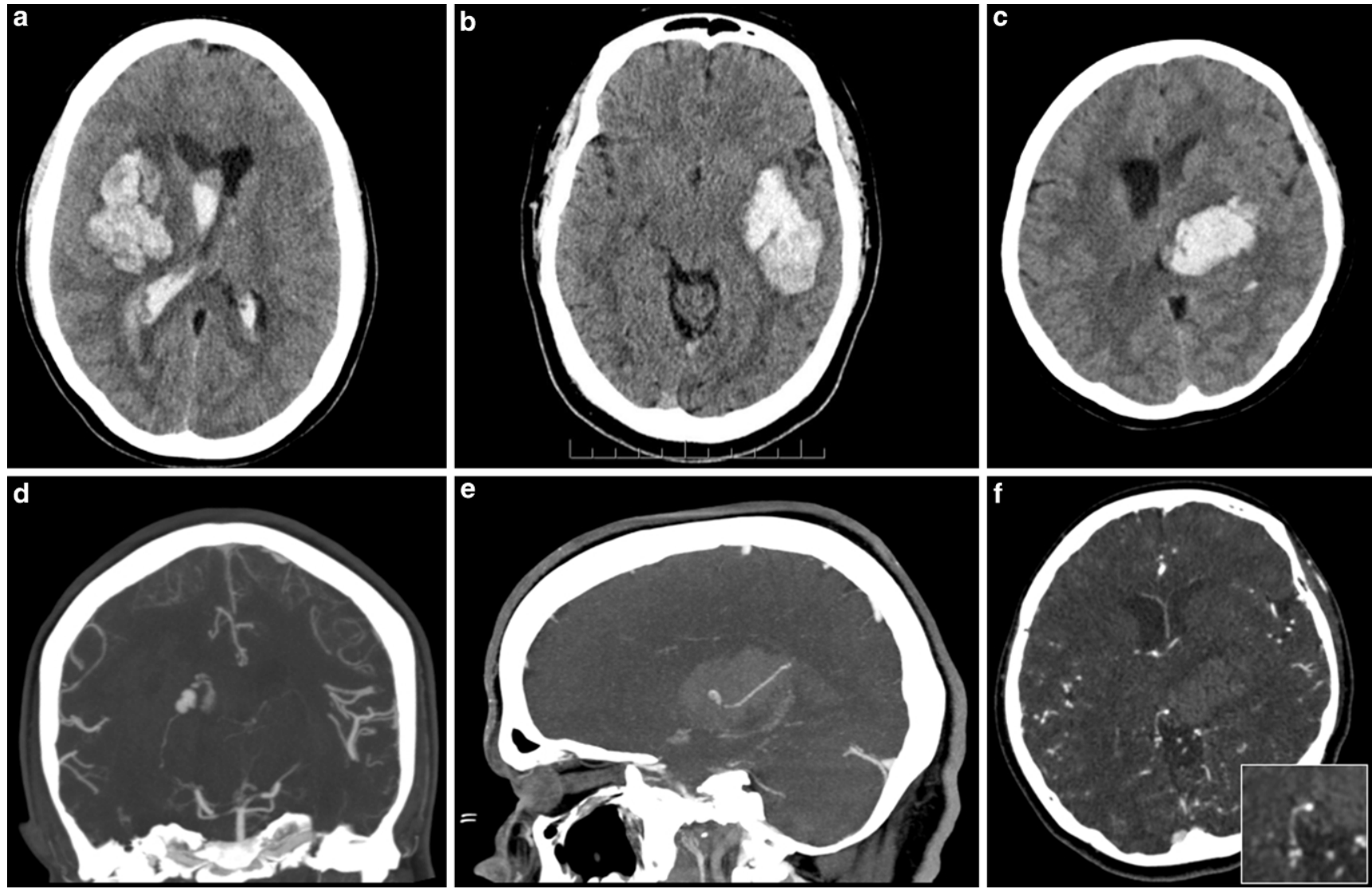

Fig. 1 Representative images from three patients: (a, d): patient 1: 30-year-old female, axial non-contrast CT (top row) showing a right frontal parenchymal hematoma with intraventricular extension. Coronal CTA (bottom row) showing a tortuous, dilated lenticulostriatal vessel coursing to the hematoma with a focal aneurysmal portion of the vessel distally, giving a spot on a string (SOAS) sign. (b, e): Patient 2: 42-year-old male, axial non-contrast CT (top row) showing a left temporal parenchymal hematoma, ventricular extension is not shown. Saggital CTA (bottom row) showing a dilated distal MCA branch coursing into the hematoma with a SOAS sign. (c, f): Patient 5: 54-year-old female, axial non-contrast CT (top row) showing a large parenchymal hematoma centered on the left thalamus. Axial CTA (bottom row) showing a SOAS sign arising from a perforator artery arising from the right posterior cerebral artery 
Fig. 2 Images from patient 3, clockwise from top left: a noncontrast CT showing a large left frontal hematoma with some layering of blood products within the hematoma. b CTA showing a left MCA branch with a SOAS sign coursing toward the hematoma. c Digital subtraction angiogram showing an aneurysmal dilatation of superior division branch of the left MCA, corresponding to the abnormal vessel seen on the CTA. d Volume-rendered 3-D reconstruction of the abnormal vessel, showing more clearly the aneurysmal "blown out" appearance of the vessel. This corresponded to the vessel wall disruption seen on the pathological specimen following surgical resection (not shown)
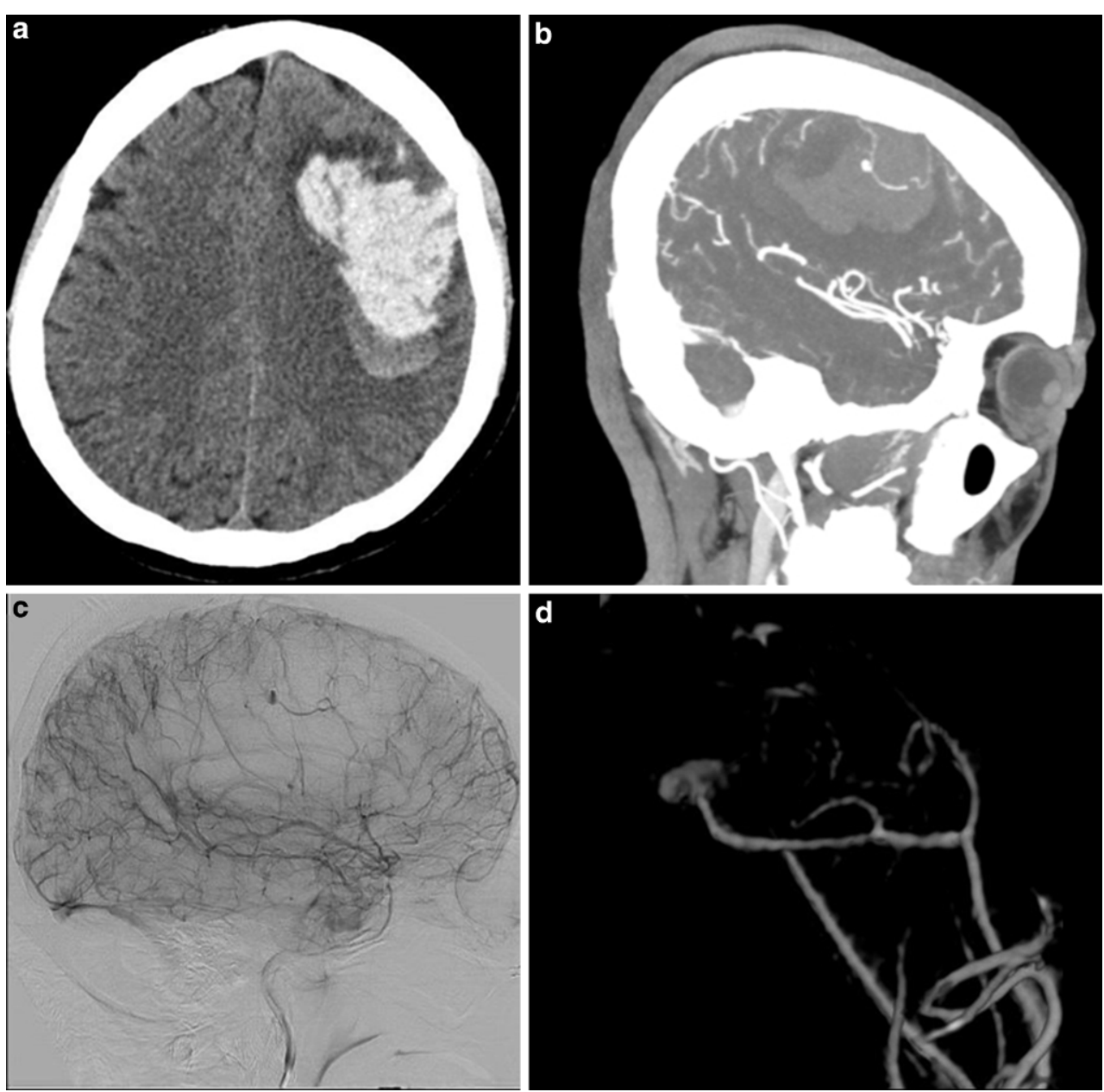

section of vessel wall with no microscopic or microbiological evidence of infection/microbial invasion. Overall, three patients were discharged to rehabilitation, three remained as inpatients and one patient died. Selected CT and DSA images are shown in Figs. 1, 2 and 3.

\section{Discussion}

\section{Why are these Findings Interesting and Distinct from the Usual Pattern seen in ICH, e.g. Hypertensive Microangiopathy Related Bleeding?}

Patients with ICH tend to be older and are more frequently on anticoagulants. Our patients were younger than the typical ICH cohort (median age 48 years), and only 1 was taking warfarin prior to admission. Furthermore, while we often see a regular spot sign in ICH patients, we have never seen a series of patients with this rare appearance of a spot on a string sign. Patients with hypertensive hemorrhages can often have normal CTA studies and they can be related to ruptured microaneurysms (Charcot-Bouchard aneurysms
[6]), which are usually not seen on a CTA. In contrast, with our patients we were able to confidently identify the source of bleeding in all cases. Furthermore, while three of our patients had a positive toxicology screening for cocaine or methamphetamine, this has not been reported to result in such an abnormal vessel. In addition, urine-based toxicology screening has a significant false positive rate [7]. These findings also raise the importance of performing a CTA on patients with a large-volume parenchymal hematoma, especially in younger patients (e.g. $<60$ years of age).

\section{Why are these Findings not just ICH Secondary to Mycotic Aneurysms?}

Given the clinical presentations, we do not think that these represent a series of mycotic or infectious aneurysms. Apart from one patient, none had any infectious endocarditis or other risk factors, and none had clinical features suggestive of active infection. Indeed, the remaining patients all had negative echocardiograms and had no evidence of systemic infection on other scans or blood tests. In addition, the spot on a string sign is not the typical imaging appear- 
Fig. 3 Images from patient 6, clockwise from top left: a noncontrast CT showing a left frontal parenchymal hematoma. There was also intraventricular extension (not shown). b Sagittal maximal intensity projection (MIP) from CTA showing a spot on a string (SOAS) sign coursing along the edge of the hematoma. c, d Left ICA injection, lateral view (c), and magnified microcatheter injection $\mathrm{s}(\mathbf{d})$ of the left callosomarginal artery in the same projection, showing the aneurysmal segment of the vessel more distally, with some contrast medium stagnation within it
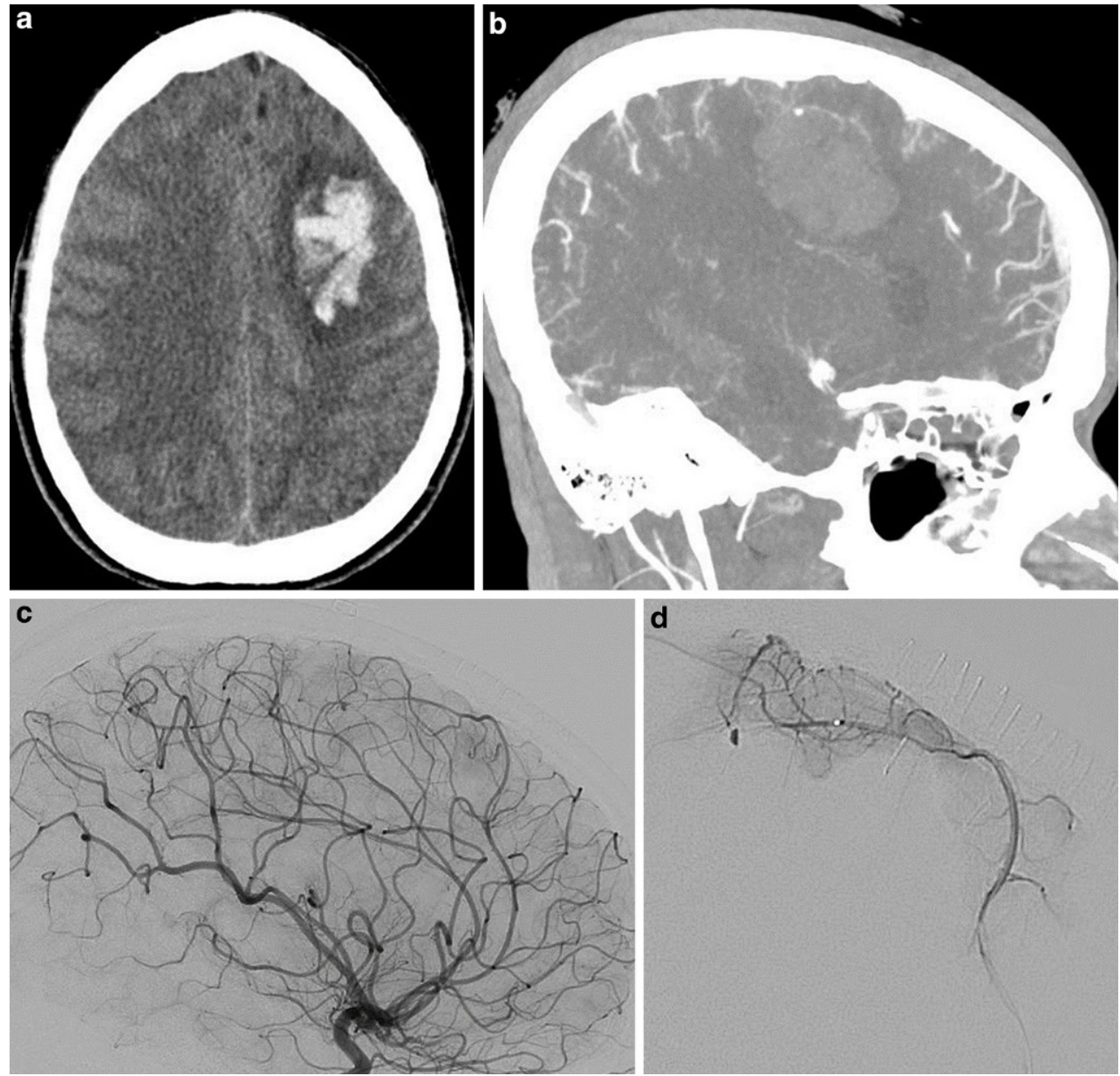

ance of mycotic aneurysms, which are often multiple and more commonly fusiform [8]. In our patients the abnormal vessels were unifocal and rather than being fusiform were comprised of an abnormal segment of vessel wall at the end of a tortuous feeding vessel that disappeared on follow-up imaging. Furthermore, in our unit (a tertiary referral neurovascular center) we see on average 1-2 mycotic aneurysms per year. It is therefore quite remarkable that all patients presented over the course of 2 months. Finally, in patient 3 who underwent surgical resection, in whom the imaging appearances were remarkably similar to the other patients in our series, the pathology showed no evidence of bacterial or neutrophilic invasion of the disrupted arterial wall.

\section{How are these Findings Different from Common Vascular Malformations?}

We certainly need to consider the atypical imaging and clinical appearances of the group overall. These patients had no evidence of a fistulous lesion (such as an AVM/dAVF) on either CTA or DSA. In addition, the clinical features were unusual. Our patients had much larger bleeds than we commonly see in ICH patients (median hematoma volume $58.4 \mathrm{~cm}^{3}$ ), especially for a cohort of patients so young, the majority of whom had no significant comorbidities. For comparison, the median hematoma volume in a summary of recent ICH trials was approximately $10 \mathrm{~cm}^{3}$ [9], while another study of 277 patients on warfarin found an average hematoma volume of $22.1 \mathrm{~cm}^{3}$. The mean patient age in these studies was 65 years and 68.5 years, respectively, another reason why our patients would be expected to have smaller hematoma volumes. One would also expect patients on anticoagulants to have much larger bleeds. For example, in one study evaluating hematoma volume according to anticoagulation type, the median ICH volume was $8.9 \mathrm{~cm}^{3}$ in patients on warfarin, far smaller than the massive bleeds in most of our cohort.

\section{Could there be an Environmental Factor in Play?}

We are reluctant to label any unusual pathology that has been admitted to our institution in the recent past as possibly related to COVID-19, but the possibility should be 
considered. One could point out that all our patients had negative COVID tests; however, the nasopharyngeal swab test has a poor sensitivity for the detection of SARS-CoV-2 [10]. Furthermore, there is emerging evidence for a systemic endotheliitis-type picture in patients with COVID-19 [11]. We also know that the ACE2 protein (which SARSCoV-2 uses to enter cells) is expressed in vascular endothelium [12]. In addition, we have not seen this vascular pattern before now. There is therefore a remote possibility that our patients are suffering from a direct effect of this virus on the cerebral vasculature, with resultant arterial disruption and massive hemorrhage. This is merely speculative, however, and merely wish to draw the attention of clinical teams to this interesting and recent phenomenon of unusual intracerebral hemorrhages in younger patients all of whom have a spot on a string sign. Note should be made that this is a single center small series of patients, and while interesting, no firm conclusions can be made at this stage.

\section{Conclusion}

We have described an unusual and rare imaging finding in a cohort of younger patients with large-volume $\mathrm{ICH}$, all of whom presented in a 2 -month period. The cause is unclear, but we encourage other clinicians to be aware of and vigilant for this phenomenon, especially in younger patients with such a bleeding pattern.

Funding This research was not supported by specific funding.

Author Contribution All authors contributed to the manuscript.

\section{Compliance with ethical guidelines}

Conflict of interest P. Nicholson, M. Gao, I. Radovanovic, V. MendesPereira, M. Hodaie, A. Pikula and T. Krings declare that they have no competing interests.
Ethical standards This study was covered by institutional Research Ethics Board approval.

\section{References}

1. Feigin VL, Lawes CM, Bennett DA, Anderson CS. Stroke epidemiology: a review of population-based studies of incidence, prevalence, and case-fatality in the late 20th century. Lancet Neurol. 2003;2:43-53.

2. Wada R, Aviv RI, Fox AJ, Sahlas DJ, Gladstone DJ, Tomlinson G, et al. CT angiography "spot sign" predicts hematoma expansion in acute intracerebral hemorrhage. Stroke. 2007;38:1257-62.

3. Charcot JM, Bouchard C. Nouvelles recherches sur la pathogénie hémor-rhagie cérébrales. Arch Physiol Norm Pathol. 1868;1:725-34

4. Sorimachi T, Osada T, Baba T, Inoue G, Atsumi H, Ishizaka H, et al. The striate artery, hematoma, and spot sign on coronal images of computed tomography angiography in putaminal intracerebral hemorrhage. Stroke. 2013;44:1830-2.

5. Kothari RU, Brott T, Broderick JP, Barsan WG, Sauerbeck LR, Zuccarello M, et al. The ABCs of measuring intracerebral hemorrhage volumes. Stroke. 1996;27:1304-5.

6. Sutherland GR, Auer RN. Primary intracerebral hemorrhage. J Clin Neurosci. 2006;13:511-7.

7. Saitman A, Park HD, Fitzgerald RL. False-positive interferences of common urine drug screen immunoassays: a review. J Anal Toxicol. 2014;38:387-96.

8. Lee WK, Mossop PJ, Little AF, Fitt GJ, Vrazas JI, Hoang JK, et al. Infected (mycotic) aneurysms: spectrum of imaging appearances and management. Radiographics. 2008;28:1853-68.

9. Yang J, Arima H, Wu G, Heeley E, Delcourt C, Zhou J, et al. Prognostic significance of perihematomal edema in acute intracerebral hemorrhage: pooled analysis from the intensive blood pressure reduction in acute cerebral hemorrhage trial studies. Stroke. 2015;46:1009-13.

10. Wang W, Xu Y, Gao R, Lu R, Han K, Wu G, et al. Detection of SARS-CoV-2 in Different Types of Clinical Specimens. JAMA. 2020;323:1843-4.

11. Varga Z, Flammer AJ, Steiger P, Haberecker M, Andermatt R, Zinkernagel AS, et al. Endothelial cell infection and endotheliitis in COVID-19. Lancet. 2020;395:1417-8.

12. Hamming I, Timens W, Bulthuis ML, Lely AT, Navis G, van Goor H. Tissue distribution of ACE2 protein, the functional receptor for SARS coronavirus. A first step in understanding SARS pathogenesis. J Pathol. 2004;203:631-7. 